\title{
The Statistical Properties of Host Load
}

\author{
Peter A. Dinda \\ July 1998 \\ CMU-CS-98-143
}

\author{
School of Computer Science \\ Carnegie Mellon University \\ Pittsburgh, PA 15213
}

A version of this paper will appear in the Proceedings of the Fourth Workshop on Languages, Compilers, and Run-time Systems for Scalable Computers (LCR98).

Effort sponsored in part by the Advanced Research Projects Agency and Rome Laboratory, Air Force Materiel Command, USAF, under agreement number F30602-96-1-0287, in part by the National Science Foundation under Grant CMS-9318163, and in part by a grant from the Intel Corporation. The U.S. Government is authorized to reproduce and distribute reprints for Governmental purposes notwithstanding any copyright annotation thereon. The views and conclusions contained herein are those of the authors and should not be interpreted as necessarily representing the official policies or endorsements, either expressed or implied, of the Advanced Research Projects Agency, Rome Laboratory, or the U.S. Government. 
Keywords: load traces, load statistics, statistical analysis, time-series analysis, self-similarity, epochal behavior 


\begin{abstract}
Understanding how host load changes over time is instrumental in predicting the execution time of tasks or jobs, such as in dynamic load balancing and distributed soft real-time systems. To improve this understanding, we collected week-long, $1 \mathrm{~Hz}$ resolution Unix load average traces on 38 different machines including production and research cluster machines, compute servers, and desktop workstations Separate sets of traces were collected at two different times of the year. The traces capture all of the dynamic load information available to user-level programs on these machines. We present a detailed statistical analysis of these traces here, including summary statistics, distributions, and time series analysis results. Two significant new results are that load is self-similar and that it displays epochal behavior. All of the traces exhibit a high degree of self similarity with Hurst parameters ranging from .63 to .97, strongly biased toward the top of that range. The traces also display epochal behavior in that the local frequency content of the load signal remains quite stable for long periods of time (150-450 seconds mean) and changes abruptly at epoch boundaries.
\end{abstract}





\section{Introduction}

The distributed computing environments to which most users have access consist of a collection of loosely interconnected hosts running vendor operating systems. Tasks are initiated independently by users and are scheduled locally by a vendor supplied operating system; there is no global scheduler that controls access to the hosts. As users run their jobs the computational load on the individual hosts changes over time.

Deciding how to map computations to hosts in systems with such dynamically changing loads (what we will call the mapping problem) is a basic problem that arises in a number of important contexts, such as dynamically load-balancing the tasks in a parallel program [20, 1, 22], and scheduling tasks to meet deadlines in a distributed soft real-time system $[12,18,19]$.

Host load has a significant effect on running time. Indeed, the running time of a compute bound task is directly related to the average load it encounters during execution. Determining a good mapping of a task requires a prediction, either implicit or explicit, of the load on the prospective remote hosts to which the task could be mapped. Making such predictions demands an understanding of the qualitative and quantitative properties of load on real systems. If the tasks to be mapped are short, this understanding of load should extend to correspondingly fine resolutions. Unfortunately, to date there has been little work on characterizing the properties of load at fine resolutions. The available studies concentrate on understanding functions of load, such as availability [17] or job durations $[6,14,8]$. Furthermore, they deal with the coarse grain behavior of load - how it changes over minutes, hours and days.

This paper is a first step to a better understanding the properties of load on real systems at fine resolutions. We collected week-long, $1 \mathrm{~Hz}$ resolution traces of the Unix load average on 38 different machines that we classify as production and research cluster machines, compute servers, or desktop workstations. We collected two sets of such traces at different times of the year. The 1 $\mathrm{Hz}$ sample rate is sufficient to capture all of the dynamic load information that is available to userlevel programs running on these machines. In this paper, we present a detailed statistical analysis of the first set of traces, taken in August, 1997. The results are similar for the second set, so we have omitted them to save space. We also contemplate the implications of those properties for the mapping problem.

The basic question is whether load traces that might seem at first glance to be random and unpredictable might have structure that could be exploited by a mapping algorithm. Our results suggest that load traces do indeed have some structure in the form of clearly identifiable properties. In essence, our results characterize how load varies, which should be of interest not only to developers of mapping and prediction algorithms, but also to those who need to generate realistic synthetic loads in simulators or to those doing analytic work. Here is a summary of our results and their implications:

(1) The traces exhibit low means but very high standard deviations and maximums. Only four traces had mean loads near 1.0. The standard deviation is typically at least as large as the mean, while the maximums can be as much as two orders of magnitude larger. The implication is that these machines have plenty of cycles to spare to execute jobs, but the execution time of these jobs will vary drastically.

(2) Standard deviation and maximum, which are absolute measures of variation, are positively correlated with the mean, so a machine with a high mean load will also tend to have a large standard 
deviation and maximum. However, these measures do not grow as quickly as the mean, so their corresponding relative measures actually shrink as the mean increases. The implication is that if the mapping problem assumes a relative metric, it may not be unreasonable to use the host with higher mean load.

(3) The traces have complex, rough, and often multimodal distributions that are not well fitted by analytic distributions such as the normal or exponential distributions. Even for the traces which exhibit unimodally distributed load, the normal distribution's tail is too short while the exponential distribution's tail is too long. The implication is that modeling and simulation that assumes convenient analytical load distributions may be flawed.

(4) Time series analysis of the traces shows that load is strongly correlated over time. The autocorrelation function typically decays very slowly while the periodogram shows a broad, almost noise-like combination of all frequency components. An important implication is that historybased load prediction schemes seem very feasible. However, the complex frequency domain behavior suggests that linear modeling schemes may have difficulty. From a modeling point of view, it is clearly important that these dependencies between successive load measurements are captured.

(5) The traces are self-similar. Their Hurst parameters range from .63 to .97 , with a strong bias toward the top of that range. This tells us that load varies in complex ways on all time scales and is long term dependent. This has several important implications. First, smoothing load by averaging over an interval results in much smaller decreases in variance than if load were not long range dependent. Variance decays with increasing interval length $m$ and Hurst parameter $H$ as $m^{2 H-2}$. This is $m^{-1.0}$ for signals without long range dependence and $m^{-0.74}$ to $m^{-0.06}$ for the range of $H$ we measured. This suggests that task migration in the face of adverse load conditions may be preferable to waiting for the adversity to be ameliorated over the long term. The self-similarity result also suggests certain modeling approaches, such as fractional ARIMA models $[9,7,3]$ which can capture this property.

(6) The traces display epochal behavior. The local frequency content of the load signal remains quite stable for long periods of time (150-450 seconds mean) and changes abruptly at the boundaries of such epochs. This suggests that the problem of predicting load may be able to be decomposed into a sequence of smaller subproblems.

\section{Measurement methodology}

The load on a Unix system at any given instant is the number of processes that are running or are ready to run, which is the length of the ready queue maintained by the scheduler. The kernel samples the length of the the ready queue at some rate and exponentially averages some number of previous samples to produce a load average which can be accessed from a user program.

We developed a small tool to sample the Digital Unix (DUX) one minute load average at one second intervals and log the resulting time series to a data file. The $1 \mathrm{~Hz}$ sample rate was arrived at by subjecting DUX systems to varying loads and sampling at progressively higher rates to determine the rate at which DUX actually updated the value. DUX updates the value at a rate of $1 / 2 \mathrm{~Hz}$, thus we chose a $1 \mathrm{~Hz}$ sample rate by the Nyquist criterion. This choice of sample rate means we capture all of the dynamic load information the operating system makes available to user programs. We ran this trace collection tool on 38 hosts belonging to the Computing, Media, and 


\begin{tabular}{l|r|r|r|r|r|r|r|r|r} 
& $\begin{array}{c}\text { Mean } \\
\text { Load }\end{array}$ & \multicolumn{1}{c|}{$\begin{array}{c}\text { Sdev } \\
\text { Load }\end{array}$} & $\begin{array}{c}\text { CoV } \\
\text { Load }\end{array}$ & $\begin{array}{c}\text { Max } \\
\text { Load }\end{array}$ & $\begin{array}{c}\text { Max/Mean } \\
\text { Load }\end{array}$ & $\begin{array}{c}\text { Mean } \\
\text { Epoch }\end{array}$ & $\begin{array}{c}\text { Sdev } \\
\text { Epoch }\end{array}$ & $\begin{array}{c}\text { COV } \\
\text { Epoch }\end{array}$ & $\begin{array}{c}\text { Hurst } \\
\text { Param }\end{array}$ \\
\hline Mean Load & 1.00 & & & & & & & & \\
\hline Sdev Load & 0.53 & 1.00 & & & & & & & \\
\hline COV Load & -0.49 & -0.22 & 1.00 & & & & & & \\
\hline Max Load & 0.60 & 0.18 & -0.32 & 1.00 & & & & & \\
\hline Max/Mean Load & -0.36 & -0.39 & 0.51 & 0.03 & 1.00 & & & & \\
\hline Mean Epoch & -0.04 & -0.10 & -0.19 & 0.08 & -0.05 & 1.00 & & & \\
\hline Sdev Epoch & -0.02 & -0.10 & -0.20 & 0.09 & -0.06 & 0.99 & 1.00 & & \\
\hline COV Epoch & 0.07 & -0.11 & -0.23 & 0.15 & -0.02 & 0.95 & 0.96 & 1.00 & \\
\hline Hurst Param & 0.45 & 0.58 & -0.21 & 0.03 & -0.49 & 0.08 & 0.10 & 0.18 & 1.00
\end{tabular}

Figure 1: Correlation coefficients (CCs) between all of the discussed statistical properties.

Communication Laboratory (CMCL) at CMU and the Pittsburgh Supercomputing Center (PSC) for slightly more than one week in late August, 1997. A second set of week-long traces was acquired on almost exactly the same set of machines in late February and early March, 1998. The results of the statistical analysis were similar for the two sets of traces. In this paper, we concentrate on the August 1997 set.

All of the hosts in the August 1997 set were DEC Alpha DUX machines, and they form four classes:

- Production Cluster: 13 hosts of the PSC's “Supercluster", including two front-end machines (axpfea, axpfeb), four interactive machines (axp0 through axp3), and seven batch machines scheduled by a DQS [13] variant (axp4 through axp10.)

- Research Cluster: eight machines in an experimental cluster in the CMCL (manchester-1 through manchester-8.)

- Compute servers: two high performance large memory machines used by the CMCL group as compute servers for simulations and the like (mojave and sahara.)

- Desktops: 15 desktop workstations owned by members of the CMCL (aphrodite through zeno.)

\section{Statistical analysis}

We analyzed the individual load traces using summary statistics, histograms, fitting of analytic distributions, and time series analysis. The picture that emerges is that load varies over a wide range in very complex ways. Load distributions are rough and frequently multimodal. Even traces with unimodal histograms are not well fitted by common analytic distributions, which have tails that are either too short or too long. Time series analysis shows that load is strongly correlated over time, but also has complex, almost noise-like frequency domain behavior.

We summarized each of our load traces in terms of our statistical measures and computed their correlations to determine how the measures are related. Figure 1 contains the results. Each cell of the table is the correlation coefficient (CC) between the row measure and the column measure, computed over the 38 load traces. We will refer back to the highlighted cells throughout the paper. 


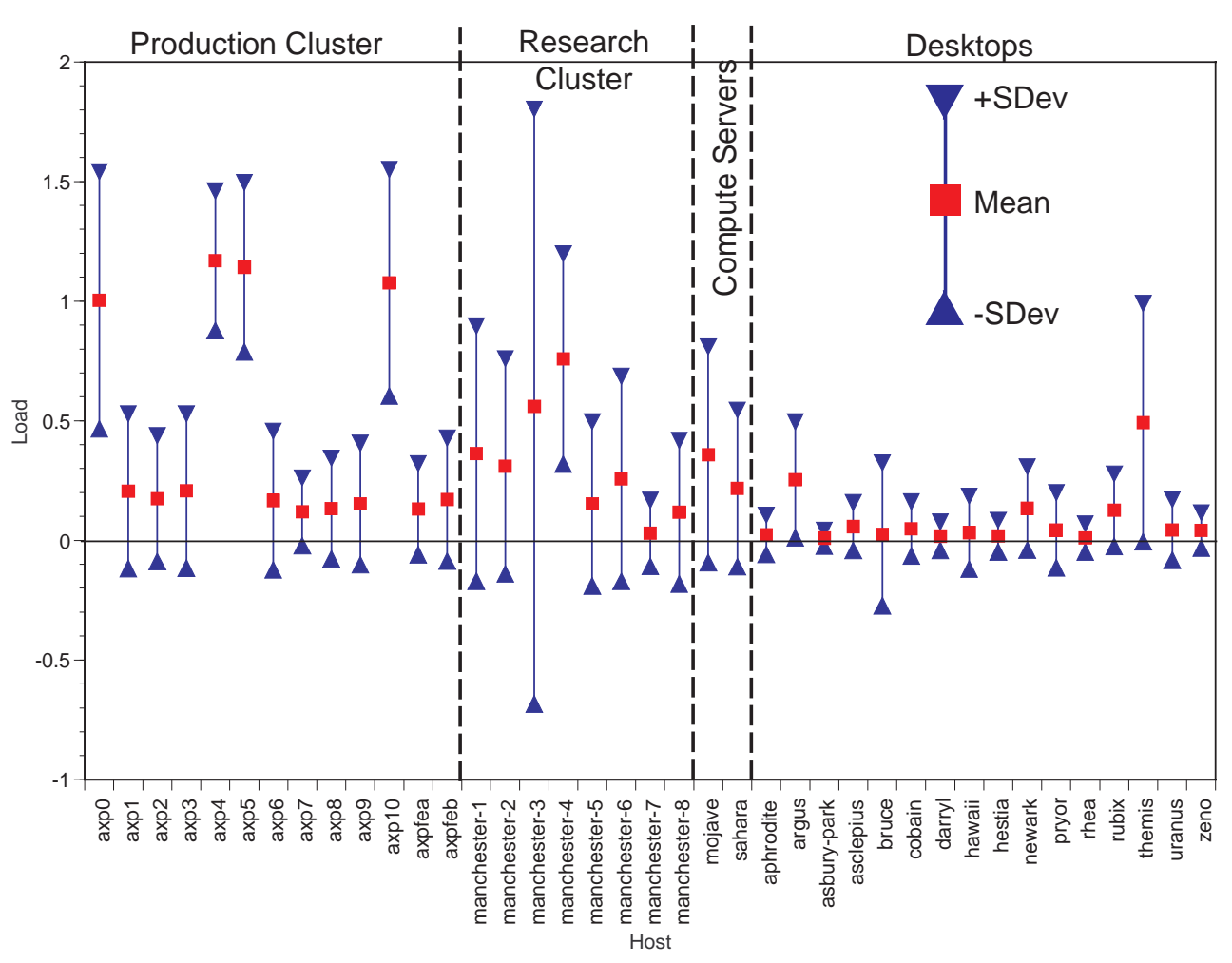

Figure 2: Mean load +/- one standard deviation.

It is important to note that these cross correlations can serve as a basis for clustering load traces into rough equivalence classes.

\section{Summary statistics:}

Summarizing each load trace in terms of its mean, standard deviation, and maximum and minimum illustrates the extent to which load varies. Figure 2 shows the mean load and the $+/$ - one standard deviation points for each of the traces. As we might expect, the mean load on desktop machines is significantly lower than on other machines. However, we can also see a lack of uniformity within each class, despite the long duration of the traces. This is most clear among the Production Cluster machines, where four machines seem to be doing most of the work. This lack of uniformity even over long time scales shows clear opportunity for load balancing or resource management systems.

From Figure 2 we can also see that desktop machines have smaller standard deviations than the other machines. Indeed, the standard deviation, which shows how much load varies in absolute terms, grows with increasing mean load $(\mathrm{CC}=0.53$ from Figure 1.) However, in relative terms, variance shrinks with increasing mean load. This can be seen in Figure 3, which plots the coefficient of variation (the standard deviation divided by the mean, abbreviated as the COV) and the mean load for each of the load traces. Here we can see that desktop machines, with their smaller mean loads, have large COVs compared to the other classes of machines. The CC between mean load and the COV of load is - 0.49 . It is clear that as load increases, it varies less in relative terms and more in absolute terms.

This difference between absolute and relative behavior also holds true for the maximum load. 


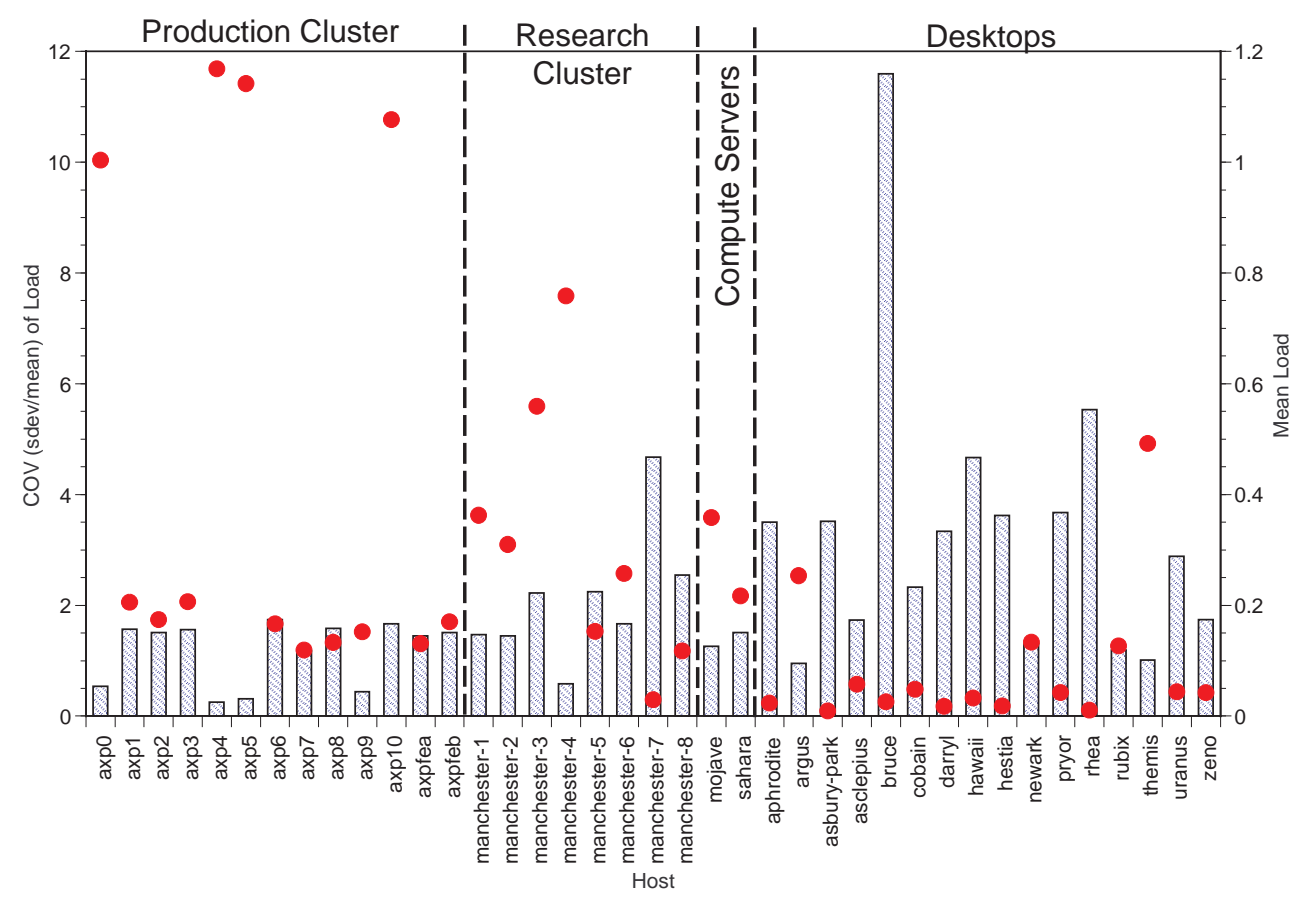

Figure 3: COV of load and mean load.

Figure 4 shows the minimum, maximum, and mean load for each of the traces. The minimum load is, not surprisingly, zero in every case. The maximum load is positively correlated with the mean load ( $\mathrm{CC}=0.60$ in Figure 1.) Figure 5 plots the ratio max/mean and the mean load for each of the traces. It is clear that this relative measure is inversely related to mean load, and Figure 1 shows that the $\mathrm{CC}$ is -0.36 . It is also important to notice that while the differences in maximum load between the hosts are rather small (Figure 4), the differences in the max/mean ratio can be quite large (Figure 5.) Desktops are clearly more surprising machines in relative terms.

With respect to the mapping problem, the implication of the differences between relative and absolute measures of variability is that lightly loaded (low mean load) hosts are not always preferable over heavily loaded hosts. For example, if the performance metric is itself a relative one (that the execution time not vary much relative to the mean execution time, say), then a more heavily loaded host may be preferable.

\section{Distributions:}

We next treated each trace as a realization of an independent, identically distributed (IID) stochastic process. Such a process is completely described by its probability distribution function (pdf), which does not change over time. Since we have a vast number of data points for each trace, histograms closely approximate this underlying pdf. We examined the histograms of each of our load traces and fitted normal and exponential distributions to them. To illustrate the following discussion, Figure 6 shows the histograms of load measurements on (a) axp0 and (b) axp7 on August 19, 1997 (86400 samples each.) Axp0 has a high mean load, while axp7 is much more lightly loaded.

Some of the traces, especially those with high mean loads, have multimodal histograms. Fig- 


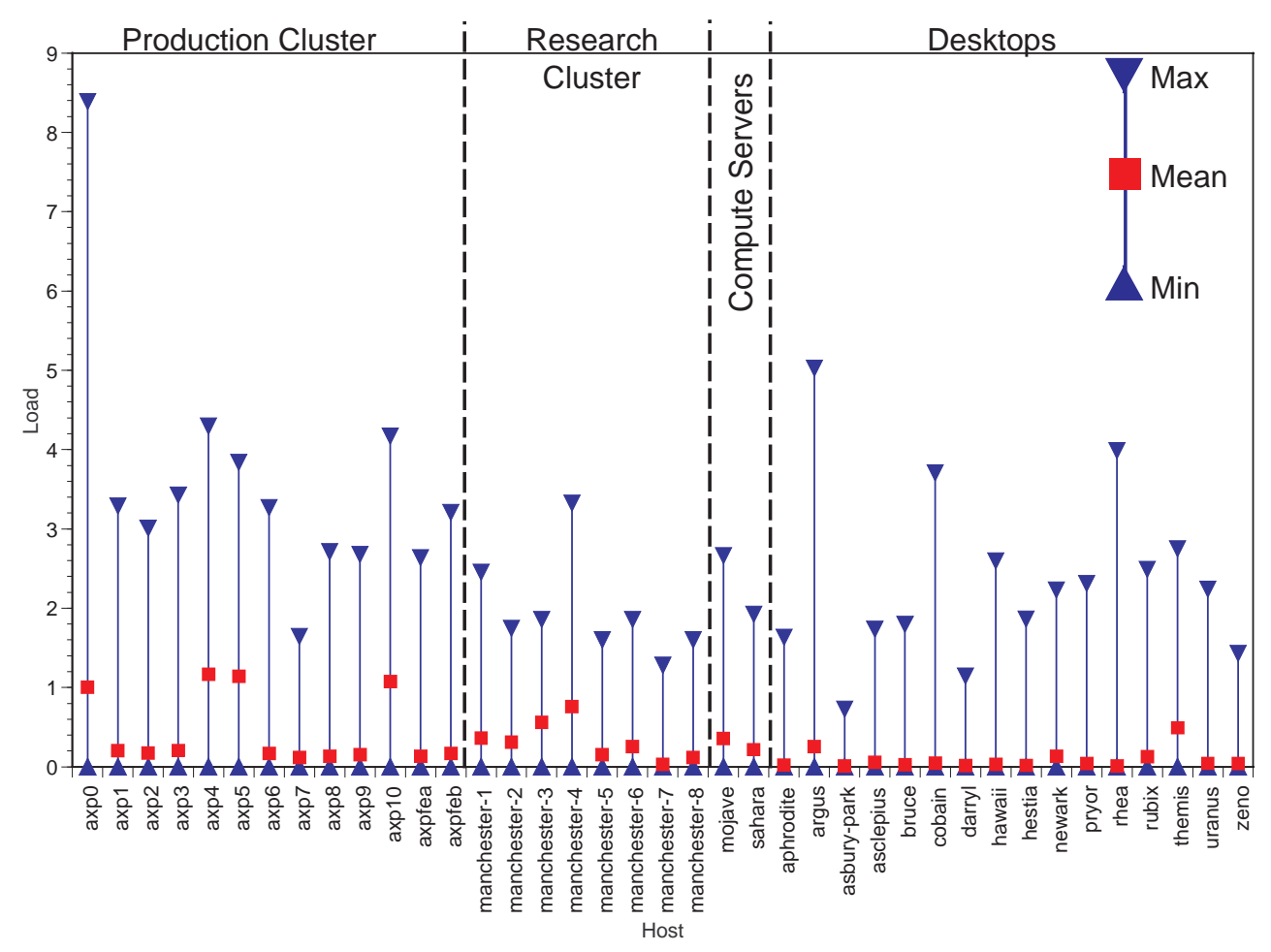

Figure 4: Minimum, maximum, and mean load.

ure 6(a) is an example of such a multimodal distribution while Figure 6(b) shows a unimodal distribution. Typically, the modes are integer multiples of 1.0 (and occasionally 0.5.) One explanation for this behavior is that jobs on these machines are for the most part compute bound and thus the ready queue length corresponds to the number of jobs. This seems plausible for the cluster machines, which run scientific workloads for the most part. However, such multimodal distributions were also noticed on the some of the other machines.

The rough appearance of the histograms (consider Figure 6(b)) is due to the fact that the underlying quantity being measured (ready queue length) is discrete. Load typically takes on 600-3000 unique values in these traces. Shannon's entropy measure [21] indicates that the load traces can be encoded in 1.4 to 8.48 bits per value, depending on the trace. These observations and the histograms suggest that load spends most of its time in one of a small number of levels.

The histograms share very few common characteristics and did not conform well to the analytic distributions we fit to them. Quantile-quantile plots are a powerful way to assess how a distribution fits data (cf. [11], pp. 196-200.) The quantiles (the $\alpha$ quantile of a pdf (or histogram) is the value $x$ at which $100 \alpha \%$ of the probability (or data) falls to the left of $x$ ) of the data set are plotted against the quantiles of the hypothetical analytic distribution. Regardless of the choice of parameters, the plot will be linear if the data fits the distribution.

We fitted normal and exponential distributions to each of the load traces. The fits are atrocious for the multimodal traces, and we do not discuss them here. For the unimodal traces, the fits are slightly better. Figure 7 shows quantile-quantile plots for (a) normal and (b) exponential distributions fitted to the unimodal axp7 load trace of Figure 6(b). Neither the normal or exponential distribution correctly captures the tails of the load traces. This can be seen in the figure. The 


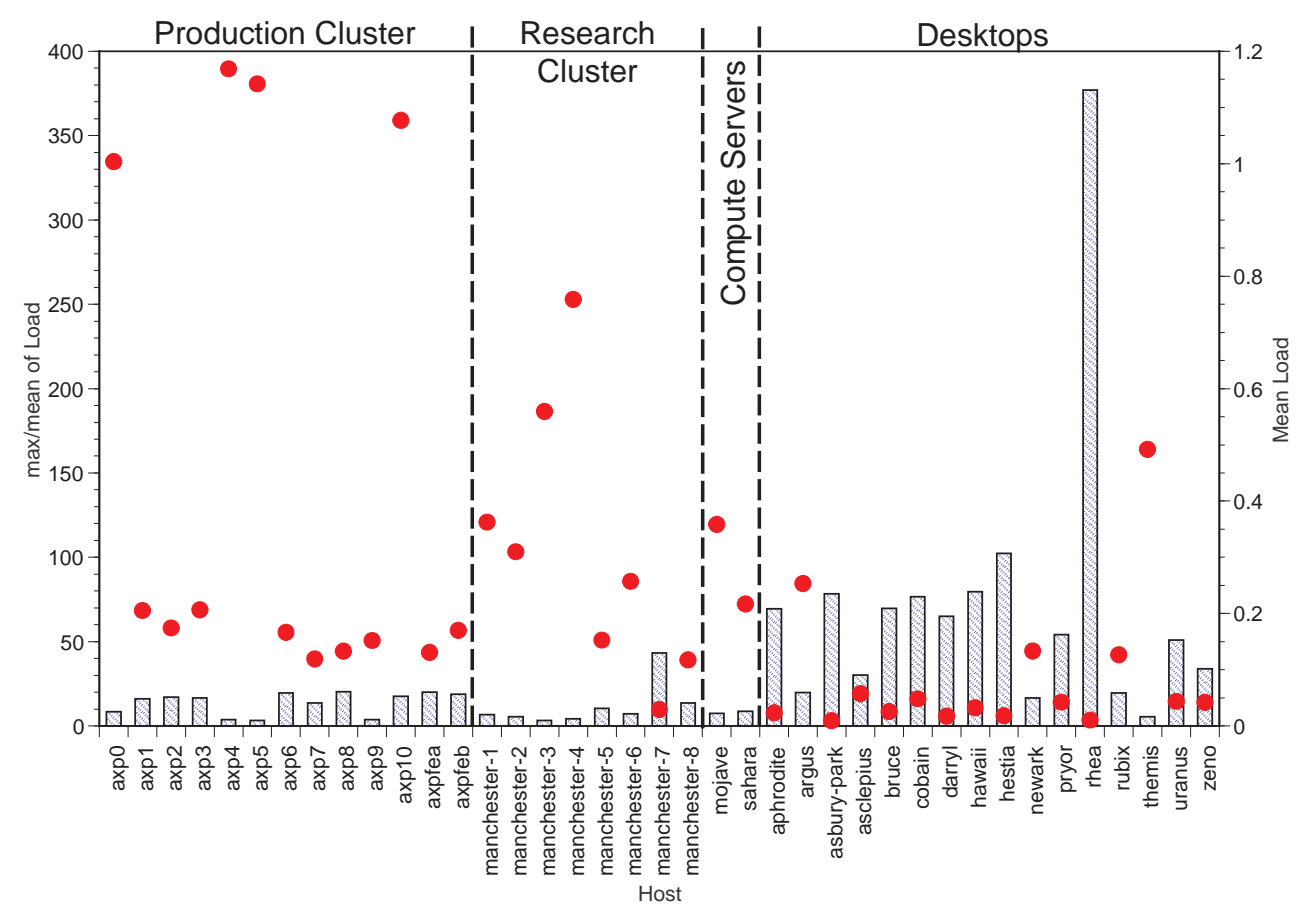

Figure 5: Maximum to mean load ratios and mean load.

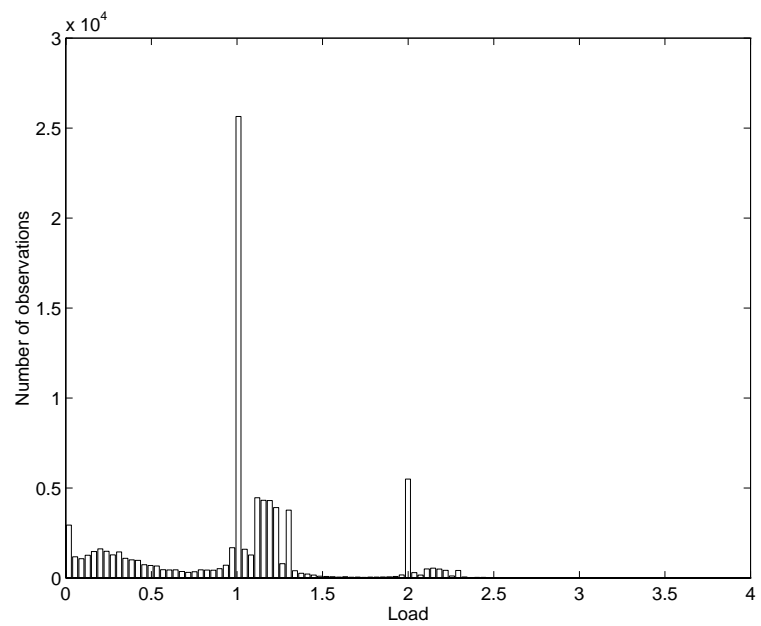

(a) $\operatorname{axp} 0$

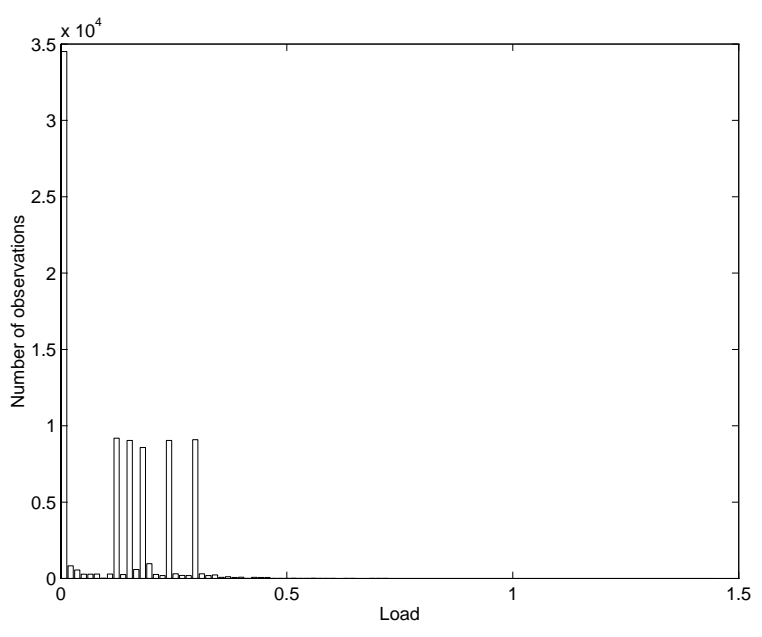

(b) $\operatorname{axp} 7$

Figure 6: Histograms for load on axp0 and axp7 on August 19, 1997.

quantiles of the data grow faster than those of the normal distribution toward the right sides of Figures 7(a). This indicates that the data has a longer or heavier tail than the normal distribution. Conversely, the quantiles of the data grow more slowly than those of the exponential distribution, as can be seen in Figures 7(b). This indicates that the data has a shorter tail than the exponential distribution. Notice that the exponential distribution goes as $e^{-x}$ while the normal distribution goes as $e^{-x^{2}}$.

There are two implications of these complex distributions. First, simulation studies and analytic 


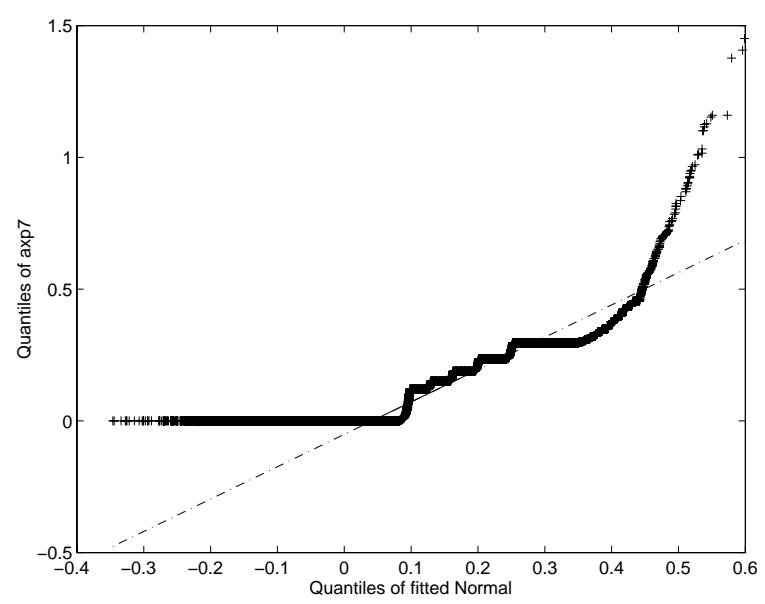

(a) $\operatorname{axp} 7$ versus normal

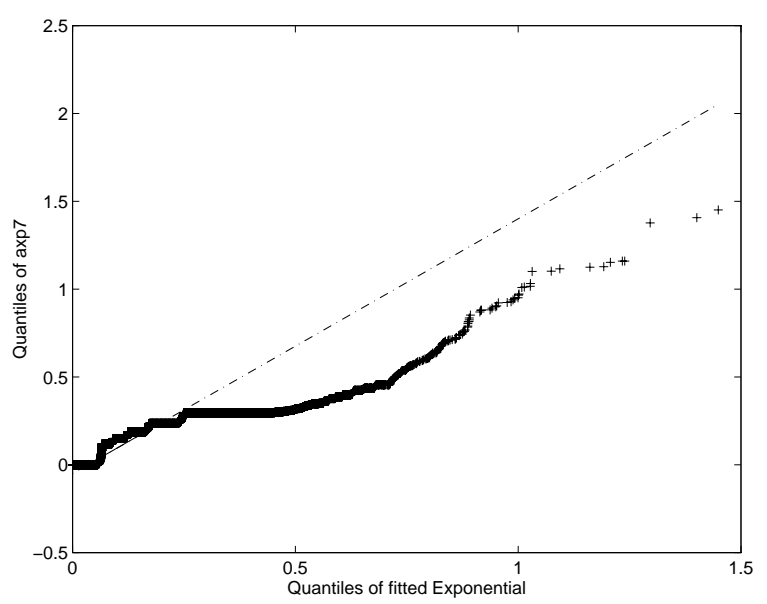

(b) $\operatorname{axp} 7$ versus exponential

Figure 7: Quantile-quantile plots for axp7 trace of August 19, 1997.

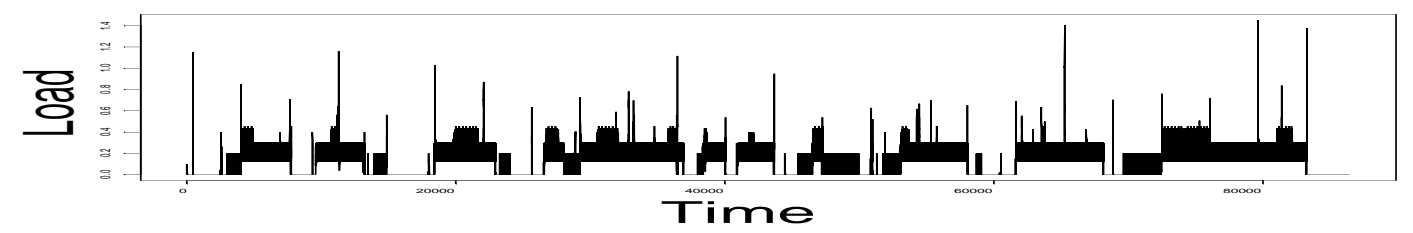

(a) Load trace for axp7 on August 19, 1997

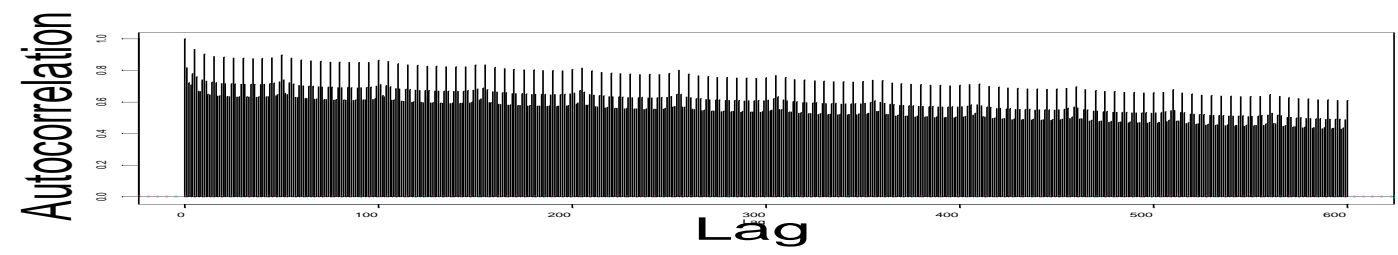

(b) Autocorrelation function of (a) to lag 600 (10 minutes)

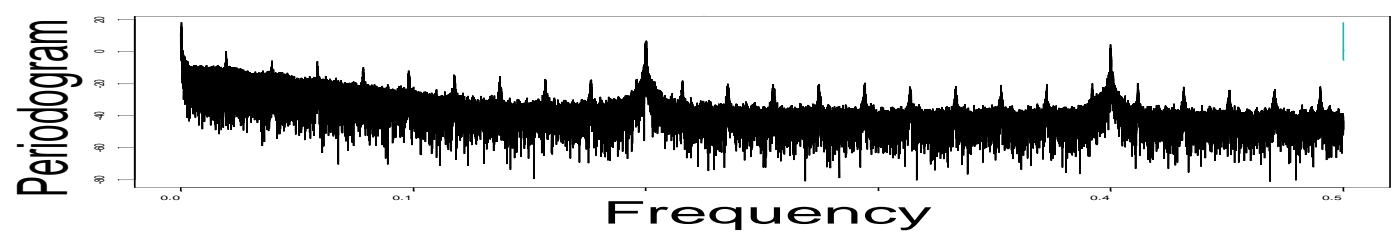

(c) Periodogram (log scale) of (a)

Figure 8: Time series analysis of axp7 load trace collected on August 19, 1997.

results predicated on simple, analytic distributions may produce erroneous results. Clearly, tracedriven simulation studies are to be preferred. The second implication is that prediction algorithms should not only reduce the overall variance of the load signal, but also produce errors that are better fit an analytic distribution. One reason for this is to make confidence intervals easier to compute. 


\section{Time series analysis:}

We examined the autocorrelation function, partial autocorrelation function, and periodogram of each of the load traces. These time series analysis tools show that past load values have a strong influence on future load values. For illustration, Figure 8 shows (a) the axp7 load trace collected on August 19, 1997, (b) its autocorrelation function to a lag of 600, and (c) its periodogram. The autocorrelation function, which ranges from -1 to 1 , shows how well a load value at time $t$ is linearly correlated with its corresponding load value at time $t+\Delta-$ in effect, how well the value at time $t$ predicts the value at time $t+\Delta$. Autocorrelation is a function of $\Delta$, and in the figure we show the results for $0 \leq \Delta \leq 600$. Notice that even at $\Delta=600$ seconds, values are still strongly correlated. This very strong, long range correlation is common to each of the load traces. For space reasons, we do not discuss the partial autocorrelation results here. However, it is important to note that the behavior of the autocorrelation and partial autocorrelation functions is instrumental to the Box-Jenkins linear time series model identification process [4].

The periodogram of a load trace is the magnitude of the Fourier transform of the load data, which we plot on a log scale (Figure 8(c).) The periodogram shows the contribution of different frequencies (horizontal axis) to the signal. What is clear in the figure, and is true of all of the load traces, is that there are significant contributions from all frequencies - the signal looks much like noise. We believe the two noticeable peaks to be artifacts of the kernel sample rate - the kernel is not sampling the length of the ready queue frequently enough to avoid aliasing. Only a few of the other traces exhibit the smaller peaks, but they all share the broad noise-like appearance of this trace.

There are several implications of this time series analysis. First, the existence of such strong autocorrelation implies that load prediction based on past load values is feasible. It also suggests that simulation models and analytical work that eschews this very clear dependence may be in error. Finally, the almost noise-like periodograms suggest that quite complex, possibly nonlinear models will be necessary to produce or predict load.

\section{Self-similarity}

The key observation of this section is that each of the load traces exhibits a high degree of selfsimilarity. This is significant for two reasons. First, it means that load varies significantly across all time-scales - it is not the case that increasing smoothing of the load quickly tames its variance. A job will have a great deal of variance in its running time regardless of how long it is. Second, it suggests that load is difficult to model and predict well. In particular, self-similarity is indicative of long memory, possibly non-stationary stochastic processes such as fractional ARIMA models [9, $7,3]$, and fitting such models to data and evaluating them can be quite expensive.

Figure 9 visually demonstrates the self similarity of the axp7 load trace. The top graph in the figure plots the load on this machine versus time for 10 days. Each subsequent graph "zooms in" on the highlighted central $25 \%$ of the previous graph, until we reach the bottom graph, which shows the central 60 seconds of the trace. The plots are scaled to make the behavior on each time scale obvious. In particular, over longer time scales, wider scales are necessary. Intuitively, a self-similar signal is one that looks similar on different time scales given this rescaling. Although the behavior on the different graphs is not identical, we can clearly see that there is significant variation on all 


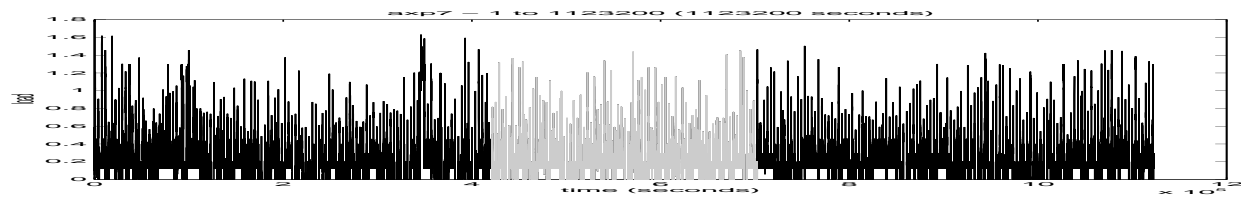

10 days

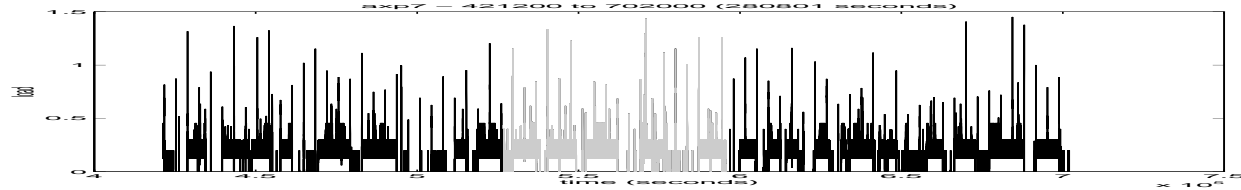

2.5 days

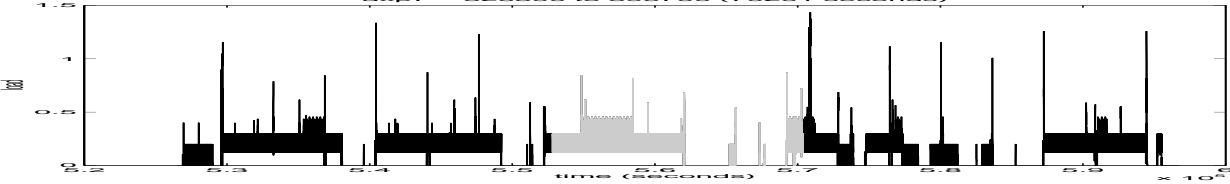

16 hours

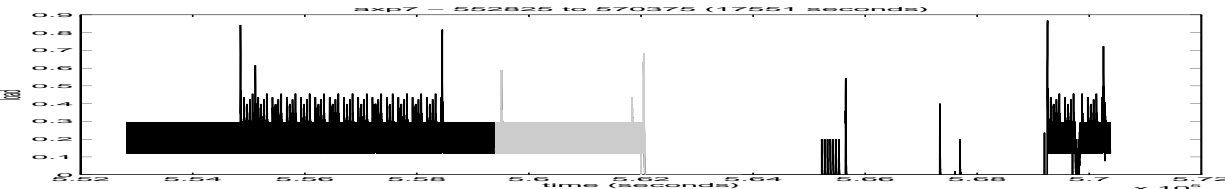

4 hours

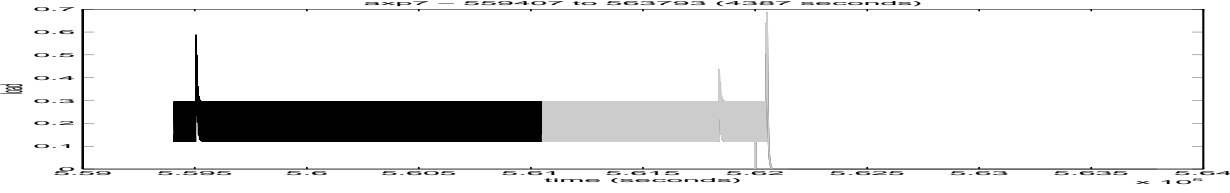

64 minutes

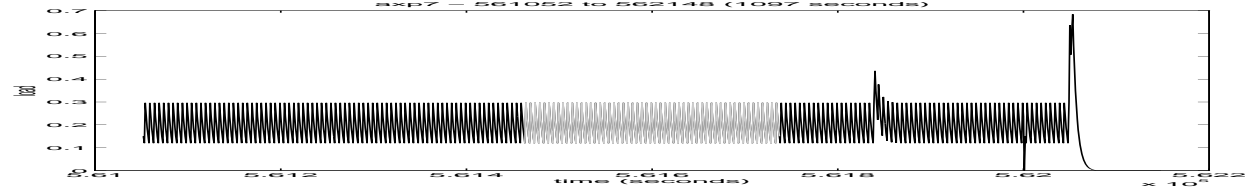

16 minutes

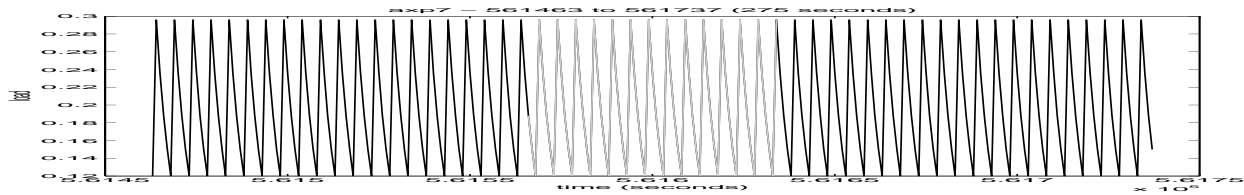

4 minutes

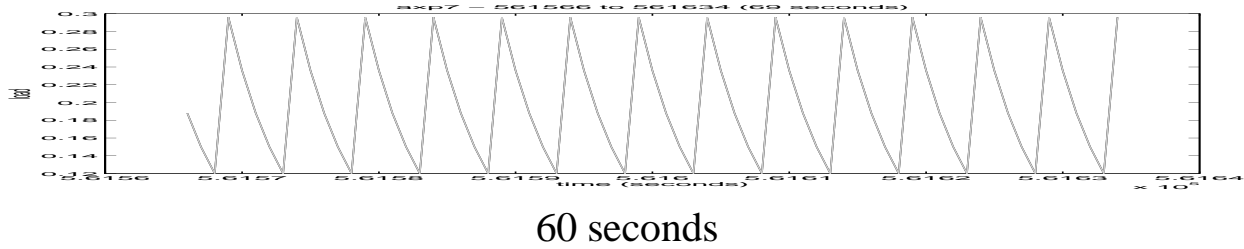

Figure 9: Visual representation of self-similarity. Each graph plots load versus time and "zooms in" on the middle quarter 


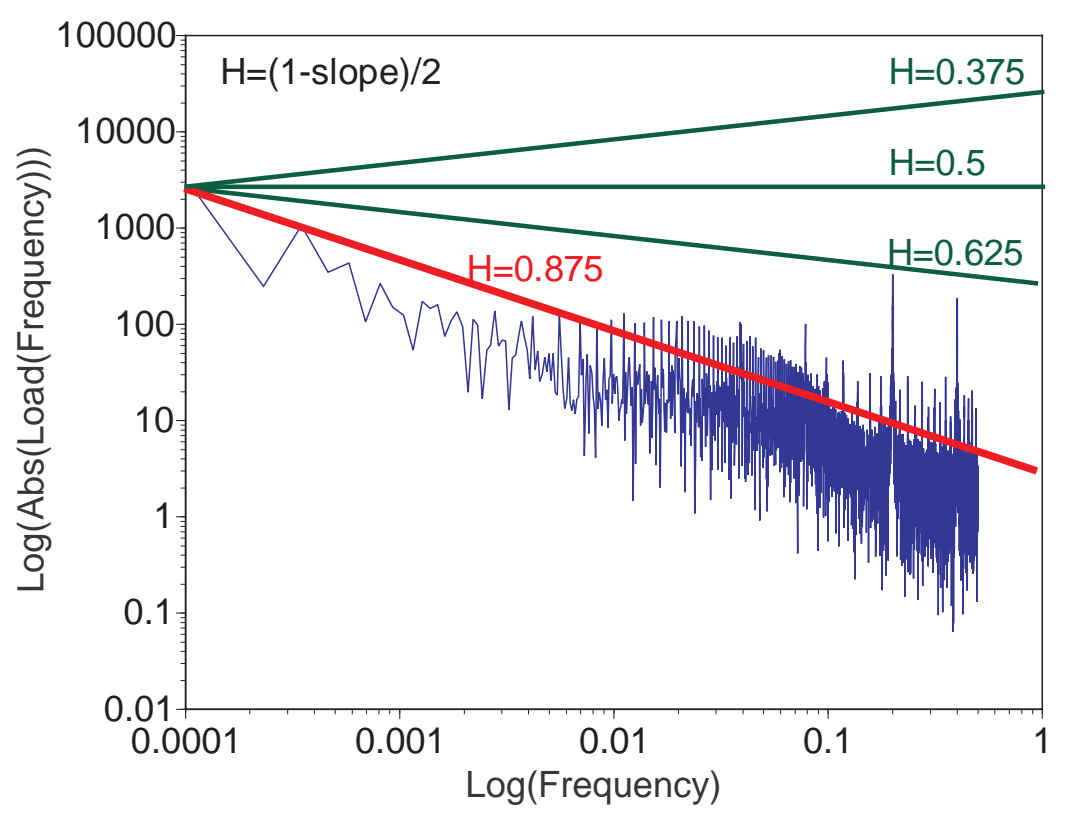

Figure 10: Meaning of the Hurst parameter in frequency domain.

time scales.

An important point is that as we smooth the signal (as we do visually as we "zoom out" toward the top of the page in Figure 9), the load signal strongly resists becoming uniform. This suggests that low frequency components are significant in the overall mix of the signal, or, equivalently, that there is significant long range dependence. It is this property of self-similar signals that most strongly differentiates them and causes significant modeling difficulty.

Self-similarity is more than intuition - it is a well defined mathematical statement about the relationship of the autocorrelation functions of increasingly smoothed versions of certain kinds of long-memory stochastic processes. These stochastic processes model the sort of the mechanisms that give rise to self-similar signals. We shall avoid a mathematical treatment here, but interested readers may want to consult [15] or [16] for a treatment in the context of networking or [2] for its connection to fractal geometry, or [3] for a treatment from a linear time series point of view.

The degree and nature of the self-similarity of a sequence is summarized by the Hurst parameter, $H$ [10]. Intuitively, $H$ describes the relative contribution of low and high frequency components to the signal. Consider Figure 10, which plots the periodogram (the magnitude of the Fourier transform) of the axp7 load trace of August 19, 1997 on a log-log scale. In this transformed form, we can describe the trend with a line of slope $-\beta$ (meaning that the periodogram decays hyperbolically with frequency $\omega$ as $\omega^{-\beta}$. The Hurst parameter $H$ is then defined as $H=(1+\beta) / 2$. As we can see in Figure 10, $H=0.5$ corresponds to a line of zero slope. This is the uncorrelated noise case, where all frequencies make a roughly equal contribution. As $H$ increases beyond 0.5 , we see that low frequencies make more of a contribution. Similarly, as $H$ decreases below 0.5 , low frequencies make less of a contribution. $H>0.5$ indicates self-similarity with positive near neighbor correlation, while $H<0.5$ indicates self-similarity with negative near neighbor correlation.

We examined each of the 38 load traces for self-similarity and estimated each one's Hurst parameter. There are many different estimators for the Hurst parameter [23], but there is no consensus 


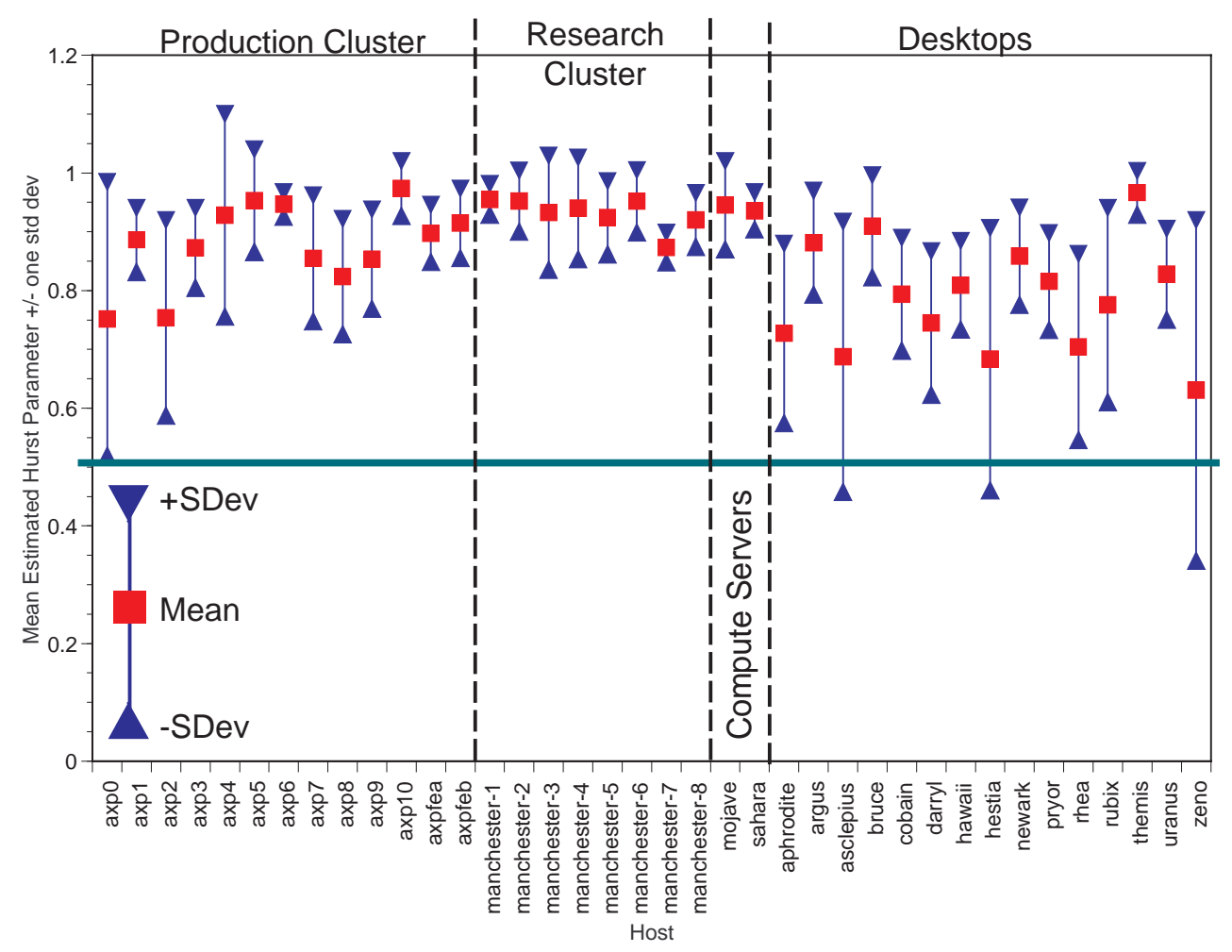

Figure 11: Mean Hurst parameter estimates for traces $+/$ - one standard deviation.

on how to best estimate the Hurst parameter of a measured series. The most common technique is to use several Hurst parameter estimators and try to find agreement among them. The four Hurst parameter estimators we used were R/S analysis, the variance-time method, dispersional analysis, and power spectral analysis. A description of these estimators as well as several others may be found in [2]. The advantage of these estimators is that they make no assumptions about the stochastic process that generated the sequence. However, they also cannot provide confidence intervals for their estimates. Estimators such as the Whittle estimator [3] can provide confidence intervals, but a stochastic process model must be assumed over which an $H$ can be found that maximizes its likelihood.

We implemented R/S analysis and the variance-time method using Matlab and performed dispersional analysis and power spectral analysis by hand on graphs prepared via Matlab. We validated each method by examining degenerate series with known $H$ and series with specific $H$ generated using the random midpoint displacement method. The dispersional analysis method was found to be rather weak for $H$ values less than about 0.8 and the power spectral analysis method gave the most consistent results.

Figure 11 presents our estimates of the Hurst parameters of each of the 38 load traces. In the graph, each central point is the mean of the four estimates, while the outlying points are at $+/$ one standard deviation. Notice that for small $H$ values, the standard deviation is high due to the inaccuracy of dispersional analysis. The important point is that the mean Hurst estimates are all significantly above the $H=0.5$ line and except for three traces with low $H$ values, their $+/$ - one standard deviation points are also above the line. 
The traces exhibit self-similarity with Hurst parameters ranging from 0.63 to 0.97 , with a strong bias toward the top of that range. On examination of the correlation coefficients (CCs) of Figure 1, we can see that the Hurst parameter has some correlation with mean load $(\mathrm{CC}=0.45)$, standard deviation of load $(\mathrm{CC}=0.58)$, and is inversely correlated with the max/mean load ratio $(\mathrm{CC}=-0.49)$. The latter seems somewhat surprising.

As we discussed above, self-similarity has implications for load modeling and for load smoothing. The long memory stochastic process models that can capture self-similarity tend to be expensive to fit to data and evaluate. Smoothing the load (by mapping large units of computations instead of small units, for example) may be misguided since variance may not decline with increasing smoothing intervals as quickly as quickly as expected. Consider smoothing load by averaging over an interval of length $m$. Without long range dependence $(H=0.5)$, variance would decay with $m$ as $m^{-1.0}$, while with long range dependence, as $m^{2 H-2}$ or $m^{-0.74}$ and $m^{-0.06}$ for the range of Hurst parameters we measured.

\section{Epochal behavior}

The key observation in this section is that while load changes in complex ways, the manner in which it changes remains relatively constant for relatively long periods of time. We refer to a period of time in which this stability holds true as an epoch. For example, the load signal could be a $0.25 \mathrm{~Hz}$ Sin wave for a minute and a $0.125 \mathrm{~Hz}$ sawtooth wave the next minute - each minute is an epoch. That these epochs exist and are long is significant because it suggests that modeling load can be simplified by modeling epochs separately from modeling the behavior within an epoch. Similarly, it suggests a two stage prediction process.

The spectrogram representation of a load trace immediately highlights the epochal behavior we discuss in this section. A spectrogram combines the frequency domain and time domain representations of a time series. It shows how the frequency domain changes locally (for a small segment of the signal) over time. For our purposes, this local frequency domain information is the "manner in which [the load] changes" to which we referred earlier. To form a spectrogram, we slide a window of length $w$ over the series, and at each offset $k$, we Fourier-transform the $w$ elements in the window to give us $w$ complex Fourier coefficients. Since our load series is real-valued, only the first $w / 2$ of these coefficients are needed. We form a plot where the $x$ coordinate is the offset $k$, the $y$ coordinate is the coefficient number, $1,2, \ldots, w / 2$ and the $z$ coordinate is the magnitude of the coefficient. To simplify presentation, we collapse to two dimensions by mapping the logarithm of the $z$ coordinate (the magnitude of the coefficient) to color. The spectrogram is basically a midpoint in the tradeoff between purely time-domain or frequency-domain representations. Along the $x$ axis we see the effects of time and along the $y$ axis we see the effects of frequency.

Figure 12 shows a representative case, a 24 hour trace from the PSC host axp7, taken on August 19,1997 . The top graph shows the time domain representation, while the bottom graph shows the corresponding spectrogram representation. What is important to note (and which occurs in all the spectrograms of all the traces) are the relatively wide vertical bands. These indicate that the frequency domain of the underlying signal stays relatively stable for long periods of time. We refer to the width of a band as the duration of that frequency epoch.

That these epochs exist can be explained by programs executing different phases, programs 

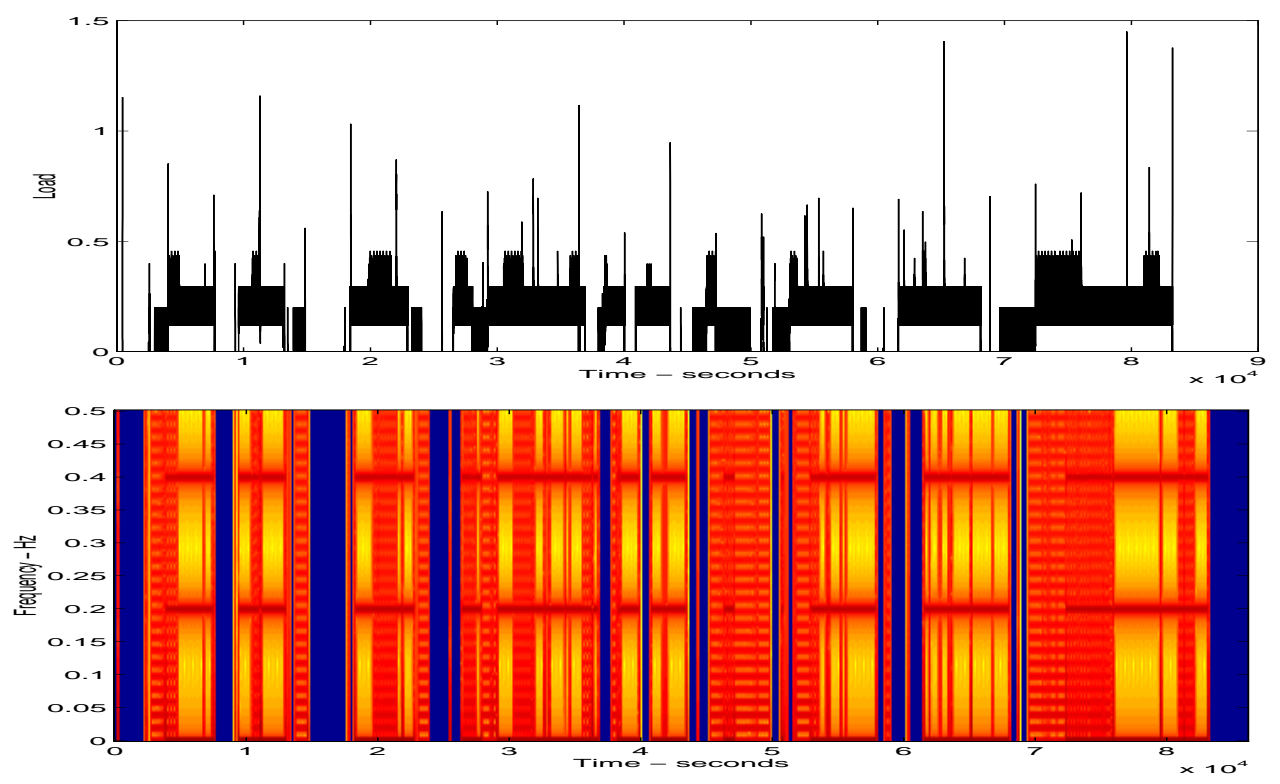

Figure 12: Time domain and spectrogram representations of load for host axp7 on August 19, 1997.

being started and shut down, and the like. The frequency content within an epoch contains energy at higher frequencies because of events that happen on smaller time-frames, such as user input, device driver execution, and daemon execution.

We can algorithmically find the edges of these epochs by computing the difference in adjacent spectra in the spectrogram and then looking for those offsets where the differences exceed a threshold. Specifically, we compute the sum of mean squared errors for each pair of adjacent spectra. The elements of this error vector are compared to an epsilon (5\% here) times the mean of the vector. Where this threshold is exceeded, a new epoch is considered to begin. Having found the epochs, we can examine their statistics. Figure 13 shows the mean epoch length and the $+/$ - one standard deviation levels for each of the load traces. The mean epoch length ranges from about 150 seconds to over 450 seconds, depending on which trace. The standard deviations are also relatively high (80 seconds to over 600 seconds.) It is the Production Cluster class which is clearly different when it comes to epoch length. The machines in this class tend to have much higher means and standard deviations than the other machines. One explanation might be that most of the machines run batch-scheduled scientific jobs which may well have longer computation phases and running times. However, two of the interactive machines also exhibit high means and standard deviations. Interestingly, there is no correlation of the mean epoch length and standard deviation to the mean load or to the Hurst parameter, as can be seen in Figure 1.

The standard deviations of epoch length in Figure 13 give us an absolute measure of the variance of epoch length. Figure 14 shows the coefficient of variance (COV) of epoch length and mean epoch length for each trace. The COV is our relative measure of epoch length variance. Unlike with load (Section 3), these absolute and relative measures of epoch length variance are both positively correlated with the mean epoch length. In addition, the correlation is especially strong ( $\mathrm{CC}=0.99$ for standard deviation and $\mathrm{CC}=0.95$ for $\mathrm{COV}$ ). As epoch length increases, it varies more in both absolute and relative terms. The statistical properties of epoch lengths are independent of 


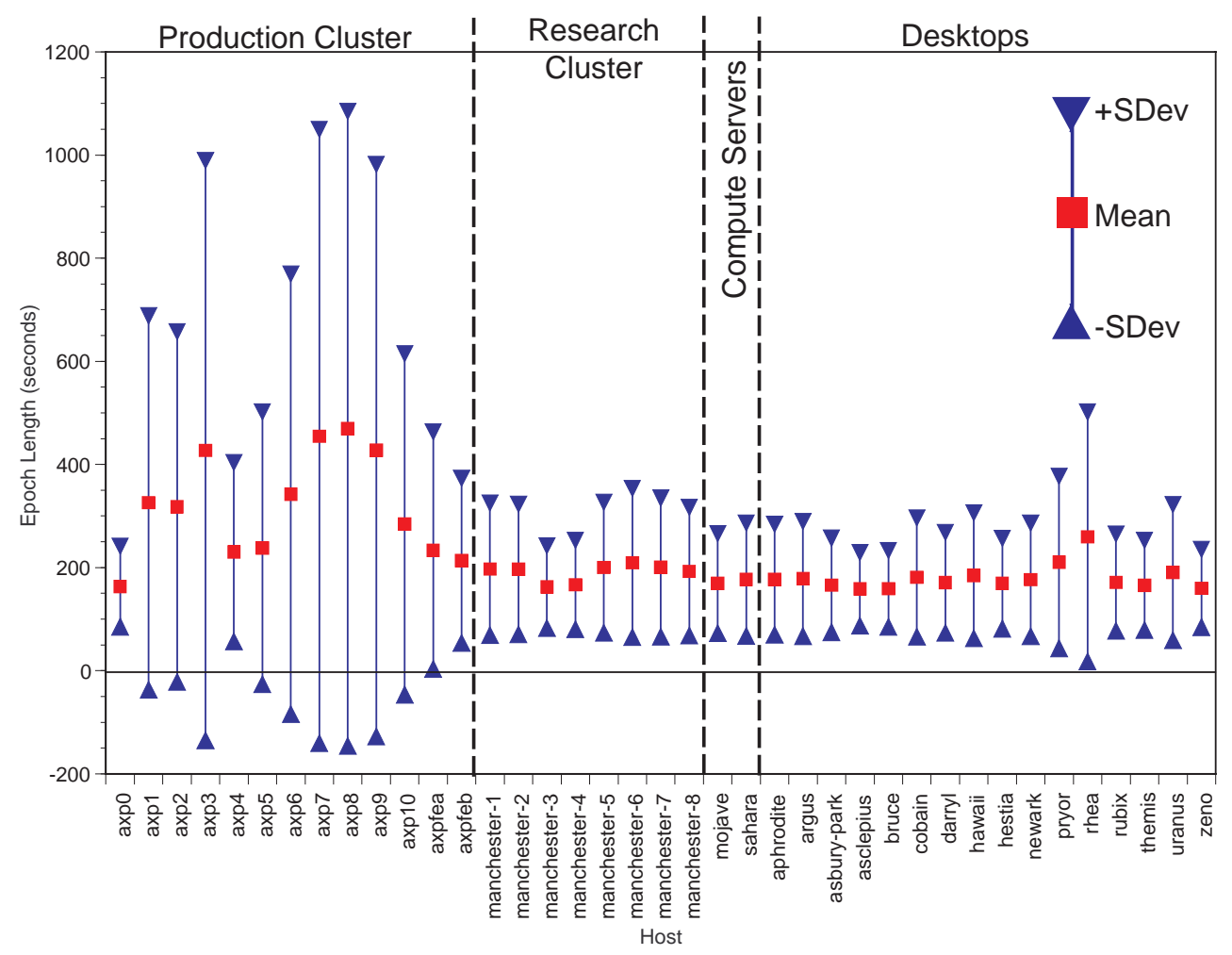

Figure 13: Mean epoch length $+/$ - one standard deviation.

the statistical properties of load.

The implication of long epoch lengths is that the problem of predicting load may be able to be decomposed into a segmentation problem (finding the epochs) and a sequence of smaller prediction subproblems (predicting load within each epoch.)

\section{Lack of seasonality:}

It is important to note that the epochal behavior of the load traces is not the same thing as seasonality in the time series analysis sense [5, 4]. Seasonality means that there are dominant (or at least visible) underlying periodic signals on top of which are layered other signals. It is not unreasonable to expect seasonality given that other studies [17] have found that availability of compute resources to change regularly over the hours of the working day and the days of the working week. However, examination of the power spectrums and autocorrelations of the load traces suggests that load does not exhibit seasonality. We feel this does not contradict the earlier results - the fluctuation of resources simply is not sufficiently periodic to qualify as seasonality in the strict time series sense.

\section{Conclusions and future work}

We collected long, fine grain load measurements on a wide variety of machines at two different times of the year. The results of an extensive statistical analysis of these traces and their implications are the following: 


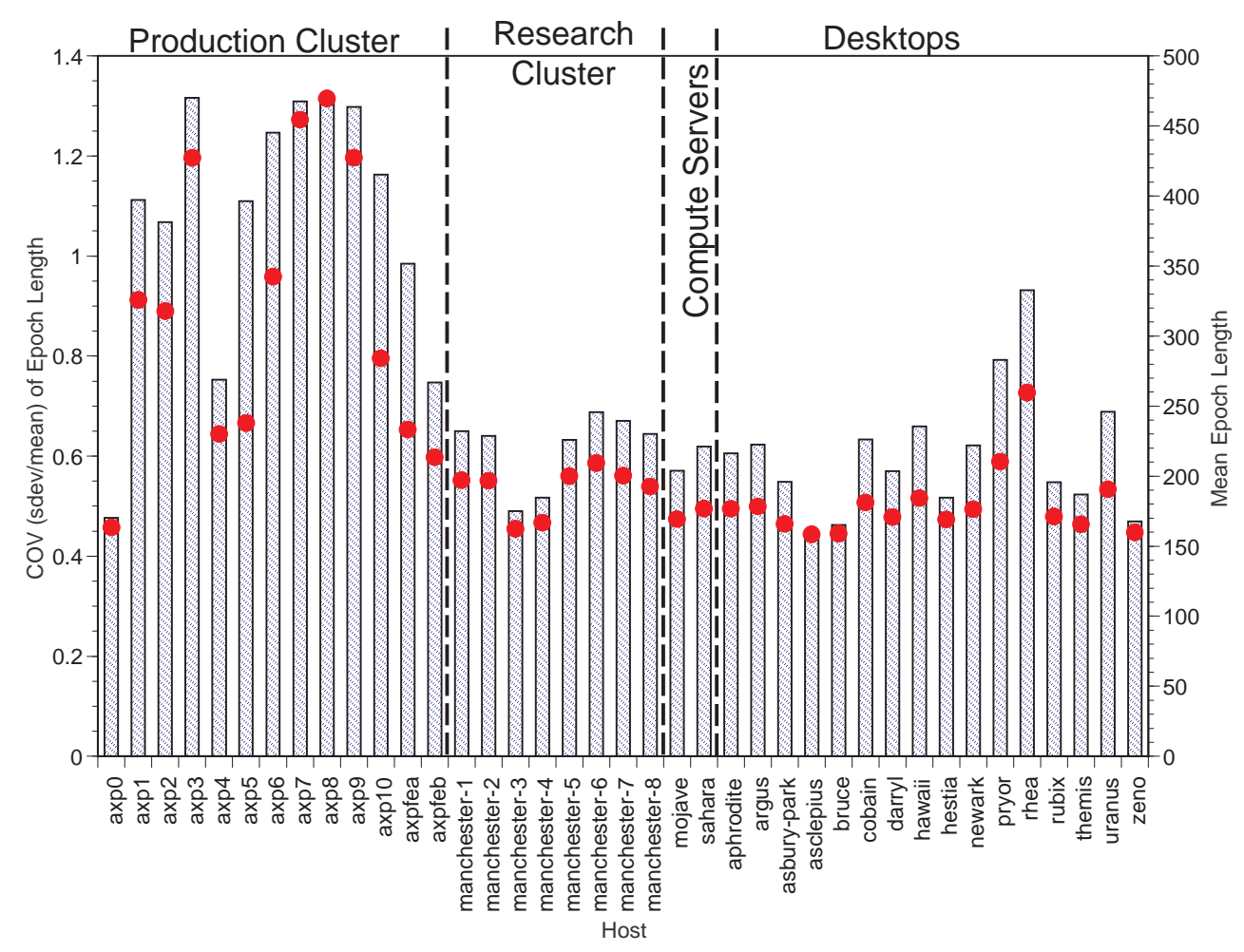

Figure 14: COV of epoch length and mean epoch length.

1. The traces exhibit low means but very high standard deviations and maximums. This implies that these machines have plenty of cycles to spare to execute jobs, but the execution time of these jobs will vary drastically.

2. Absolute measures of variation are positively correlated with the mean while relative measures are negatively correlated. This suggests that it may not be unreasonable to map tasks to heavily loaded machines under some performance metrics.

3. The traces have complex, rough, and often multimodal distributions that are not well fitted by analytic distributions such as the normal or exponential distributions, which are particularly inept at capturing the tail of the distribution. This implies that modeling and simulation that assumes convenient analytical load distributions may be flawed. Trace-driven simulation may be preferable.

4. Load is strongly correlated over time, but has a broad, almost noise-like frequency spectrum. This implies that history-based load prediction schemes are feasible, but that linear methods may have difficulty. Realistic load models should capture this dependence, or trace-driven simulation should be used.

5. The traces are self-similar with relatively high Hurst parameters. This means that load smoothing will decrease variance much more slowly than expected. It may be preferable to migrate tasks in the face of adverse load conditions instead of waiting for the adversity to be ameliorated over the long term. Self-similarity also suggests certain modeling approaches 
such as fractional ARIMA models $[9,7,3]$ and non-linear models which can capture the self similarity property.

6. The traces display epochal behavior in that the local frequency content of the load signal remains quite stable for long periods of time and changes abruptly at the boundaries of such epochs. This suggests that the problem of predicting load may be able to be decomposed into a sequence of smaller subproblems.

We are currently exploring how well the hierarchy of linear time series models [4] perform for short term prediction of load. Part of this work involves quantifying the benefit of capturing the self-similarity of load using fractional ARIMA models [7, 9, 3]. Initial results show that fractional models provide as much as a $40 \%$ improvement in prediction error and rarely perform worse than their non-fractional counterparts.

The context of our work is dynamically mapping real-time tasks to hosts in a distributed environment. In this context, we are interested in load models for classifying environments and hosts, synthesizing realistic execution times in a simulator, and for predicting execution times on remote hosts. We will gladly share the load traces we have collected with members of the research community. 


\section{Bibliography}

[1] Arabe, J., Beguelin, A., Lowekamp, B., E. Seligman, M. S., And Stephan, P. Dome: Parallel programming in a heterogeneous multi-user environment. Tech. Rep. CMUCS-95-137, Carnegie Mellon University, School of Computer Science, April 1995.

[2] Bassingthwaighte, J. B., Beard, D. A., Percival, D. B., And Raymond, G. M. Fractal structures and processes. In Chaos and the Changing Nature of Science and Medicine: An Introduction (April 1995), D. E. Herbert, Ed., no. 376 in AIP Conference Proceedings, American Institute of Physics, pp. 54-79.

[3] Beran, J. Statistical methods for data with long-range dependence. Statistical Science 7, 4 (1992), 404-427.

[4] Box, G. E. P., Jenkins, G. M., And Reinsel, G. Time Series Analysis: Forecasting and Control, 3rd ed. Prentice Hall, 1994.

[5] Brockwell, P. J., AND Davis, R. A. Introduction to Time Series and Forecasting. Springer-Verlag, 1996.

[6] Eager, D. L., Lazowska, E. D., And Zahorjan, J. The limited performance benefits of migrating active processes for load sharing. In SIGMETRICS '88 (May 1988), pp. 63-72.

[7] Granger, C. W. J., And Joyeux, R. An introduction to long-memory time series models and fractional differencing. Journal of Time Series Analysis 1, 1 (1980), 15-29.

[8] Harchol-Balter, M., And Downey, A. B. Exploiting process lifetime distributions for dynamic load balancing. In Proceedings of ACM SIGMETRICS '96 (May 1996), pp. 13-24.

[9] Hosking, J. R. M. Fractional differencing. Biometrika 68, 1 (1981), 165-76.

[10] Hurst, H. E. Long-term storage capacity of reservoirs. Transactions of the American Society of Civil Engineers 116 (1951), 770-808.

[11] JAIn, R. The Art of Computer Systems Performance Analysis. John Wiley and Sons, Inc., 1991.

[12] JENSEN, E. D. A real-time manifesto. http://www.realtime-os.com, 1996.

[13] Kaplan, J. A., And Nelson, M. L. A comparison of queueing, cluster, and distributed computing systems. Tech. Rep. NASA TM 109025 (Revision 1), NASA Langley Research Center, June 1994. 
[14] Leland, W. E., AND OtT, T. J. Load-balancing heuristics and process behavior. In Proceedings of Performance and ACM SIGMETRICS (1986), vol. 14, pp. 54-69.

[15] Leland, W. E., Taqqu, M. S., Willinger, W., And Wilson, D. V. On the self-similar nature of ethernet traffic. In Proceedings of ACM SIGCOMM'93 (September 1993).

[16] Morin, P. R. The impact of self-similarity on network performance analysis. Tech. Rep. Computer Science 95.495, Carleton University, December 1995.

[17] MutKa, M. W., And Livny, M. The available capacity of a privately owned workstation environment. Performance Evaluation 12, 4 (July 1991), 269-284.

[18] Object Management Group. Realtime corba: A white paper. http://www.omg.org, December 1996. In Progess.

[19] Polze, A., Fohler, G., And Werner, M. Predictable network computing. In Proceedings of the 17th International Conference on Distributed Computing Systems (ICDCS '97) (May 1997), pp. 423-431.

[20] Rinard, M., Scales, D., And Lam, M. Jade: A high-level machine-independent language for parallel programming. IEEE Computer 26, 6 (June 1993), 28-38.

[21] Shannon, C. E. A mathematical theory of communication. Bell System Tech. J. 27 (1948), 379-423, 623-656.

[22] Siegell, B., And SteEnkiste, P. Automatic generation of parallel programs with dynamic load balancing. In Proceedings of the Third International Symposium on HighPerformance Distributed Computing (August 1994).

[23] Taqqu, M. S., Teverovsky, V., And Willinger, W. Estimators for long-range dependence: An empirical study. Fractals 3, 4 (1995), 785-798. 